\title{
Análisis de la evolución del clima y los pastizales naturales en el noroeste de la provincia de Chubut, Argentina, durante el período 2000-2014: identificación de variables asociadas a la disminución de las existencias ganaderas en la región
}

García Martínez, G.C.; G. Ciari, J. Gaitan, C. Caruso, N. Nagahama, W. Opazo, V. Nakamatsu, C. Lloyd, C. Cotut, G. Irisarri y J. Escobar

\begin{abstract}
RESUMEN
Una elevada proporción del territorio argentino corresponde a tierras secas y una tercera parte de éstas se hallan en la Patagonia. Esta región históricamente ha sufrido procesos de deterioro del pastizal. Generalmente, la degradación es atribuida al uso ganadero; sin embargo, el efecto del clima sobre estos sistemas ha sido poco analizado. El objetivo de este trabajo fue analizar la evolución del clima y de los pastizales naturales, a través de la identificación de variables que permitan comprender los posibles cambios en las existencias ganaderas para el noroeste de la provincia de Chubut, en el período 20002014. Para ello se analizaron diferentes tipos de datos: climáticos, índice verde normalizado (NDVI), lecturas de monitores MARAS y existencias ganaderas. Los resultados indican incrementos de temperatura y tendencias negativas de la productividad y estructura del pastizal. Asimismo se observó una disminución de las existencias ganaderas. El incremento de las temperaturas podría estar relacionado con balances hídricos desfavorables, los cuales, combinados con el sobrepastoreo, pueden ser factores claves en los procesos de deterioro del pastizal. Resulta fundamental continuar con el monitoreo de las diferentes variables que caracterizan a estos sistemas y diseñar estrategias que permitan mitigar los procesos descriptos.
\end{abstract}

Palabras clave: ganadería, imágenes satelitales, monitores ambientales (MARAS), Patagonia

García Martínez, G.C.; G. Ciari, J. Gaitan, C. Caruso, N. Nagahama, W. Opazo, V. Nakamatsu, C. Lloyd, C. Cotut, G. Irisarri and J. Escobar 
2017. Analysis of the evolution of climate and natural grasslands in northwest Chubut province, Argentina, between 2000 and 2014: identification of variables associated with livestock decrease in the region. Agriscientia 34: 59-69

\section{SUMMARY}

Most of Argentina territory is classified as dry lands, one third of them is located in Patagonia. The grasslands in this region have been historically affected by deterioration. This degradation has been associated to excessive grazing. However, little attention has been paid to the effect of climate on these systems. The aim of this work was to examine the evolution of climate and grasslands in NW Chubut between 2000-2014, identifying variables that allow a better understanding of the causes of changes in livestock numbers. Different types of data have been analysed: weather statistics, normalized difference vegetation index (NDVI), information from MARAS monitors and livestock numbers. Results show a temperature increase and negative trends of grasslands productivity and structure during the period. Additionally, a decline in the livestock numbers was observed. Rising temperatures could negatively affect hydric balance which, along with overgrazing, could be key factors affecting grassland deterioration. It is of fundamental importance to keep monitoring these variables and to devise mitigation strategies against the processes described.

Key words: Animal husbandry, remote sensing, ambiental monitors (MARAS), Patagonia.

G.C. García Martínez, G. Ciari, C. Caruso, W. Opazo, C. Lloyd y C. Cotut: INTA EEA Esquel, Chacabuco 513, Esquel, Chubut, Argentina. J. Gaitan: Instituto de Suelos INTA Castelar, Instituto de Suelos. N. Nagahama: CONICET, INTA EEA Esquel. V. Nakamatsu, J. Escobar: INTA EEA Chubut. G. Irisarri: Laboratorio de Análisis Regional y Teledetección (LART), C. Forrajicultura, IFEVA, Facultad de Agronomía, Universidad de Buenos Aires-CONICET. Correspondencia a: garcia.guillermo@inta.gob.ar

\section{INTRODUCCIÓN}

Las tierras secas (zonas áridas, semiáridas y subhúmedas-secas) cubren aproximadamente el $41 \%$ de la superficie del planeta Tierra y allí reside más del 38\% de la población mundial. Debido a las restricciones climáticas, sólo el 25\% de las tierras secas del mundo están dedicadas a la agricultura; sin embargo, son de vital importancia para la ganadería, ya que el $65 \%$ son utilizadas para el pastoreo de ganado doméstico sobre la vegetación natural (Millennium Ecosystem Assessment, 2005). En la Argentina aproximadamente el $75 \%$ del territorio corresponde a tierras secas; de ellas, una tercera parte se hallan en la región patagónica. La principal actividad agropecuaria en la Patagonia es la ganadería extensiva, donde los pastizales naturales son utilizados como fuente de forraje para el ganado doméstico (Golluscio et al., 1998), por lo que su estado y tendencia resulta fundamental en términos de sustentabilidad y desarrollo. Históricamente, los pastizales naturales en gran parte de la Patagonia han sufrido procesos de deterioro (Escobar, 1997; Perelman et al., 1997; Bertiller et al., 2002; Golluscio et al., 2009), con graves implicancias en la producción secundaria. Dichos cambios han sido atribuidos principalmente al uso ganadero (Soriano, 1956a; Ares et al., 1990; Perelman et al., 1997; Oñatibia \& Aguiar, 2016). Sin embargo, el efecto climático ha sido escasamente considerado en estos análisis. Una de las razones es la falta de monitoreo de las condiciones meteorológicas y el estado de la vegetación, de forma continua y extendida en grandes superficies. Es posible que muchos de los cambios observados en estos sistemas ocurran en respuesta a actividades humanas y también a cambios en las variables ambientales (Brown et al., 1997).

En los últimos años se han desarrollado 
metodologías para el monitoreo de tierras basadas en el uso de índices de vegetación, derivados de sensores remotos, como estimadores de atributos estructurales y funcionales de los ecosistemas. Entre estos índices, el índice verde normalizado (NDVI) (Tucker, 1979) es uno de los más ampliamente utilizados, dado que en diversos estudios se han encontrado relaciones significativas entre este índice y aspectos claves de la estructura y funcionalidad de los ecosistemas, como la biomasa (Tucker et al., 1985), el índice de área foliar (Turner et al., 1999), la productividad primaria neta aérea (Paruelo et al., 1997) y la cobertura vegetal (Gaitán et al., 2013). El uso de este tipo de información permitió determinar la existencia, en los últimos años, de signos de deterioro en la vegetación en diferentes zonas del hemisferio sur (Zhao \& Running, 2010) e inclusive de la Patagonia (Gaitán et al., 2015). Varios estudios han mostrado que la integral del NDVI (NDVI-I) es un buen indicador de la productividad primaria neta aérea (PPNA) en los pastizales naturales de regiones áridas y semiáridas (Paruelo et al., 1997; Jobbágy et al., 2002).

En la última década se han instalado en la Patagonia 350 sitios de monitoreo a largo plazo del suelo y la vegetación, denominados Monitores Ambientales para Regiones Áridas y Semiáridas (MARAS) (Oliva et al., 2009; Oliva et al., 2011). Estos monitores permiten evaluar la evolución del pastizal a través de variables biofísicas como la cobertura del suelo, la estructura de parches y la estabilidad/función de los interparches, con una frecuencia de relectura de 5 años. Recientemente, en el noroeste de la provincia de Chubut, se han realizado las primeras relecturas de MARAS luego de su instalación inicial en el año 2008. Diversos trabajos han descripto la pérdida de receptividad ganadera en los pastizales patagónicos y la consecuente caída de la carga animal a lo largo del siglo pasado (Escobar, 1997; Golluscio et al., 1998); sin embargo, los análisis para períodos más recientes son escasos. La combinación de diferentes fuentes de información sobre la evolución del clima, los pastizales y las existencias ganaderas resulta importante para comprender a los sistemas de una manera integral que considere gran parte de la complejidad que abarcan.

El presente trabajo tiene por objetivo analizar conjuntamente la evolución del clima y de los pastizales naturales, identificando variables que permitan comprender mejor los posibles cambios en las existencias ganaderas para el noroeste de la provincia de Chubut, en el período 2000-2014.

\section{MATERIALES Y METODOS}

\section{Área de estudio}

El área de estudio abarca parte de los distritos Patagónico Subandino, Patagónico Occidental y Patagónico Central de la Provincia Fitogeográfica Patagónica (Soriano, 1956b). El clima es templado a templado frío, con vientos dominantes del oeste. Las precipitaciones poseen un régimen estacional, concentrándose entre otoño e invierno (Paruelo et al., 1998a; Paruelo et al., 1998b). El relieve, la circulación general de la atmósfera y la influencia de la masa polar marítima del océano Pacífico determinan un fuerte gradiente de precipitaciones oeste-este (Barros et al., 1979). Esta condición determina, a su vez, un gradiente de tipos de vegetación desde bosques hasta semidesiertos, atravesando la estepa graminosa, arbustivagraminosa y arbustiva (Soriano, 1956b). Los detritos glaciales y los materiales volcánicos son los materiales parentales más importantes de los suelos patagónicos. En las porciones occidentales más húmedas y frías pueden desarrollarse suelos mólicos; hacia el este y con el aumento de la aridez, los suelos de los órdenes Aridisol y Entisol dominan el paisaje. Éstos suelen presentar una gruesa capa calcárea cementada entre los 40 y 50 $\mathrm{cm}$ de profundidad (Del Valle, 1998).

El área de estudio abarca cuatro departamentos del NO de Chubut: Cushamen, Futaleufú, Languiñeo y Tehuelches (Figura 1). Entre los cuatro suman el $49,68 \%$ de los establecimientos ganaderos de la provincia y cuentan con el $29 \%$ de las existencias ovinas, el $67 \%$ de las bovinas y $57 \%$ de las caprinas. A su vez, el stock ganadero ovino representa el $9 \%$ del total nacional. Por otro lado, el $2 \%$ de la población vive en forma directa de la producción agropecuaria y otro tanto de servicios relacionados. El sector ganadero ovino en Chubut sostiene alrededor de 3500 establecimientos sin tener en cuenta los valles bajo riego. Si bien la actividad no representa una proporción elevada del total de la actividad económica provincial, posee gran implicancia geopolítica, por su presencia en todo el territorio, con especial énfasis en los pueblos del interior de la provincia, donde genera impactos sociales importantes (Iglesias, 2013).

\section{Variables analizadas}

Para realizar este trabajo se analizaron cuatro grupos de variables diferentes: climáticas, NDVI, monitores de vegetación MARAS y existencias ganaderas. Dada la diferente escala espacial y temporal entre cada una de las variables, y con 
el objetivo de evitar la pérdida de información valiosa, se definieron algunos criterios al momento de los análisis. En lo temporal se decidió analizar la ventana de tiempo más amplia de cada variable en el período 2000-2014. Si bien para algunas variables no se disponía de la información de todos los años entre el 2000 y el 2014, se utilizó la mayor cantidad de datos disponibles en dicho período. En lo espacial, para cada variable se realizaron análisis de cada una de las unidades mínimas (estación meteorológica, MARA, pixel) y posteriormente se integró la información regionalmente obteniendo tasas de cambio relativas promedio para el área de estudio o porcentaje de unidades (estación meteorológica, MARA, pixel) que mostraron tendencias positivas, negativas o neutras.

\section{Climáticas}

Las variables climáticas evaluadas fueron: precipitación anual (pp) y temperatura media anual (tma) de 13 estaciones meteorológicas distribuidas en el área de estudio. De las 13 estaciones, 7 disponían de datos de precipitación y temperatura, 5 solo precipitación y 1 solo temperatura. Se evaluó la tendencia de estas variables entre los años 2000 y 2014.

NDVI-I de la serie 2000-2014 a partir de datos de MODIS

Se analizó la tendencia de la integral anual del índice NDVI (NDVI-I) del sensor MODIS (producto MOD13Q1) para el período 20002014. La metodología de procesamiento de las imágenes satelitales ha sido descripta por Gaitán et al. (2015): para cada pixel se obtuvo NDVI-I de las 14 temporadas comprendidas entre 20002001 y 2013-2014, considerando la temporada de crecimiento entre el 1 de julio del año $n$ y el 30 de junio del año $n+1$. El período considerado para obtener la NDVI-I está vinculado a la fuerte estacionalidad del crecimiento de la vegetación en la región determinado por la dinámica de precipitación y temperatura (Fabricante et al., 2009; Gaitan et al., 2015). Posteriormente se realizaron regresiones entre el tiempo (temporadas de crecimiento) y la NDVI-I para cada pixel, y se construyeron mapas de tendencias.

\section{MARAS}

En los sectores áridos y semiáridos de la región el cambio en la estructura de la vegetación se analizó entre los años 2008 y 2014 a través de 22 monitores MARAS (Oliva et al., 2009; Oliva et al., 2011; Gaitán et al., 2013), ubicados en campos de productores a partir de los cuales se analizaron indicadores de vegetación tales como tamaño de los parches de vegetación (TP) y los porcentajes de: cobertura vegetal $(\mathrm{CV})$, cobertura forrajera (CF), muerto en pie (MP) y mantillo (M). El tamaño de parches (TP) es un indicador de estructura del paisaje capaz de detectar cambios de corto plazo, al igual que los demás indicadores de atributos de la vegetación como cobertura vegetal (CV), cobertura forrajera (CF), muerto en pie (MP) y mantillo (M) (Oliva et al., 2009; Oliva et al., 2011). Para la instalación de los monitores MARAS se colocaron y demarcaron con estacas fijas tres transectas de $50 \mathrm{~m}$ de longitud. Cada MARAS fue releída dos veces con un intervalo de 5 años y respetando la ubicación exacta de las transectas. En dos de las transectas se realizaron mediciones de cobertura vegetal a través del método de intercepción de puntos (Muller-Dombois \& Ellenberg, 1974). En cada una de esas transectas se registró el tipo de intercepción cada $20 \mathrm{~cm}$ con el objetivo de obtener información de cobertura vegetal $(\mathrm{CV})$, cobertura forrajera (CF), muerto en pie (MP) y mantillo (M). En la tercera transecta se midió el tamaño de parches de vegetación (TP), entre otras variables.

Para evaluar el porcentaje de cambio anual de estos indicadores estructurales se aplicó la siguiente fórmula:

\section{(valor final - valor inicial) $* 100$ valor inicial $* N^{\circ}$ años}

\section{Existencias ganaderas}

Las existencias ganaderas se obtuvieron a partir de la información provista por la Dirección General de Estadísticas y Censos con los datos de la "Encuesta ganadera anual provincial". Cabe aclarar que no se dispone de datos para todos los años de la serie. Debido a que el 2005 es el primer año posterior al 2000 del cual se poseen datos, se analizó el período 2005-2013. Se transformaron los datos de cada especie animal a unidades ganaderas ovinas según el siguiente criterio: 1 ovino = 1 UGO (Unidad Ganadera Ovina); 1 bovino $=6,3$ UGO; 1 equino = 7,5 UGO; 1 caprino $=1$ UGO (Escobar, 1997). Se realizó la sumatoria de UGO de todas las especies animales consideradas en el censo de los cuatro departamentos de la que se obtuvo un único valor por temporada.

\section{Análisis de datos}

Las tendencias de las variables climáticas, NDVI-I y existencias ganaderas fueron analizadas mediante regresión lineal, donde se consideraron como variables dependientes la PP, TMA, UGOs y NDVI-I y como variable independiente, los años. La 
pendiente de dicha relación es un descriptor de la velocidad de cambio anual. Para las variables climáticas y de existencias ganaderas, se evaluó la significancia de la regresión observando los límites de confianza de la distribución de datos. Para el NDVI-I, en cambio, a partir del signo de la pendiente y de la significancia estadística del coeficiente de regresión se clasificaron las tendencias de los pixeles en tres grupos: píxeles con tendencia significativa negativa (coeficiente de correlación $<-0,53)$, tendencia significativa positiva (coeficiente de correlación > 0,53) y sin tendencia significativa (coeficiente de correlación entre -0,53 y 0,53) para el período 2000 a 2014 y para los cuatro departamentos del NO de Chubut (Gaitán et al., 2015). Los límites se establecieron según los valores críticos del coeficiente de correlación de Pearson para el nivel de significancia de 0,05 y 14 estaciones de crecimiento (12 GL). A su vez, se calcularon las tasas de disminución o aumento del NDVI-I, lo cual indicaría diferentes velocidades de ocurrencia de procesos de deterioro (tasas más negativas) o recuperación (tasas más positivas). Para ello se calculó la pendiente de la regresión entre los años y NDVI-I y se expresó como porcentaje del valor inicial de la serie (temporada de crecimiento 2000-2001).

\section{Análisis complementarios}

Tanto para el clima como para la carga ganadera contamos con datos previos al año 2000. Del total de estaciones meteorológicas, nueve disponen de datos desde 1960, una desde 1964 y una desde 1973. En el caso de la carga se dispone de 16 años de datos desde 1937. Se analizó la tendencia de estas variables para el período comprendido entre el inicio de cada una de estas series y el año 2014. La inclusión de un período más prolongado, aunque solo sea para algunas variables (clima y existencias ganaderas), permitió interpretar de forma más adecuada lo que ocurrió en el período más reciente (2000-2014). Del mismo modo permitió comprender mejor en qué medida los cambios observados entre el 2000 y el 2014 son parte de una tendencia histórica o producto de un patrón más reciente.

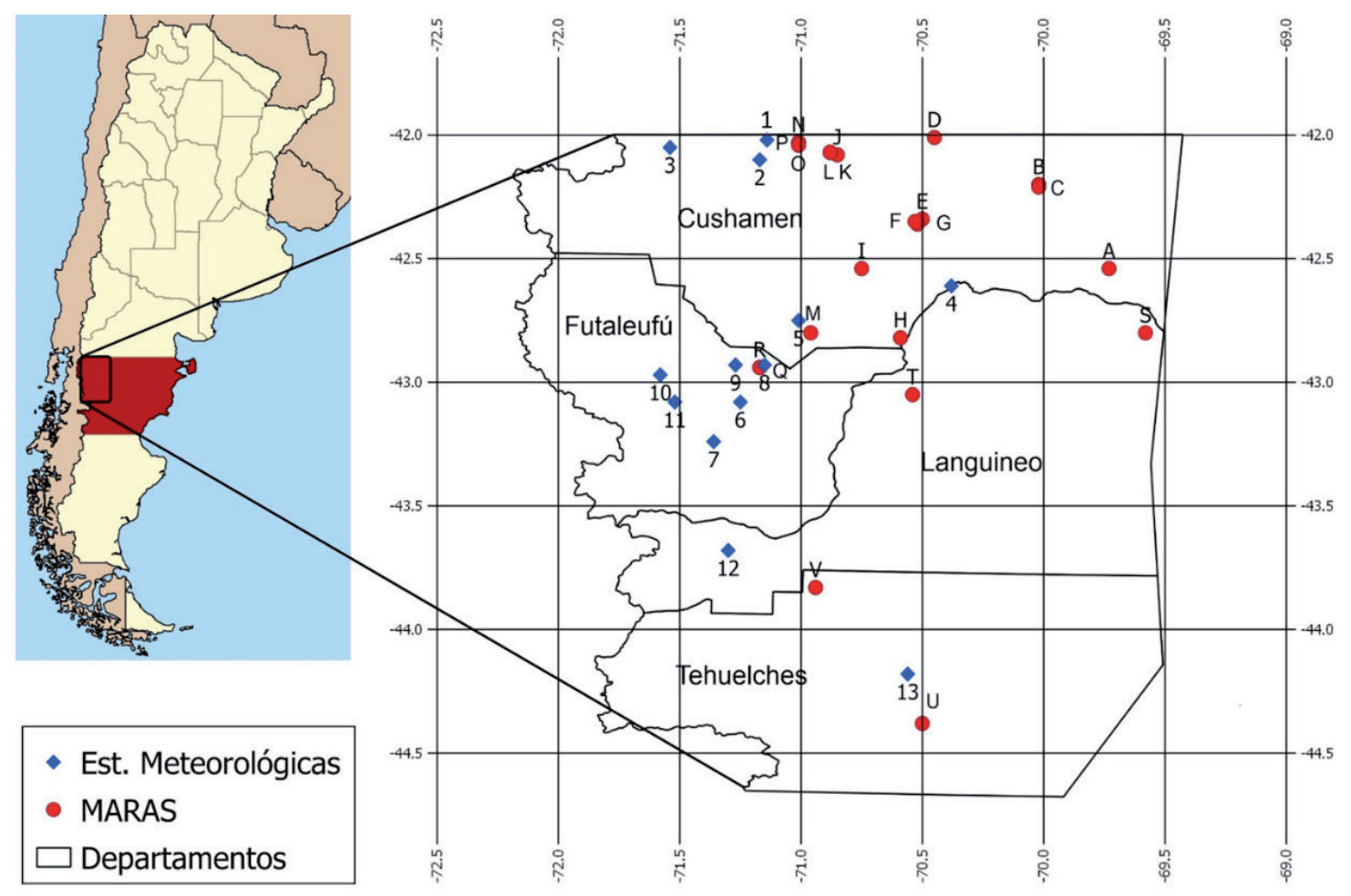

Figura 1: Ubicación de los cuatro departamentos, estaciones meteorológicas y MARAS considerados. Se indica el número o letra correspondiente a la identificación de las estaciones meteorológicas o MARAS respectivamente, en coincidencia con las tablas 1 y 2. Datos elaborados para la presente edición. 


\section{RESULTADOSY DISCUSIÓN}

El presente trabajo permitió abordar de una manera más integral la problemática del deterioro de los pastizales en el noroeste del Chubut. Se presentaron datos sobre las tendencias observadas para diferentes grupos de variables como el clima, la NDVI-I, los monitores de vegetación MARAS y las existencias ganaderas. Dada la diversidad de variables y de las escalas temporales y espaciales de cada una de ellas, solo es posible realizar un diagnóstico inicial y es necesario ser prudentes en la interpretación de los resultados. Asimismo, en futuros trabajos sería enriquecedor indagar sobre los vínculos entre las variables analizadas y otras posibles variables a considerar.

\section{Tendencia climática}

Entre el año 2000 y 2014 la mayor parte de las estaciones no mostraron tendencias significativas en cuanto a las precipitaciones. Se observó una estación con tendencia significativa positiva y una negativa (Tabla 1). En lo que respecta a la temperatura se observan incrementos significativos en seis de las ocho estaciones analizadas. Dichos incrementos tuvieron un rango entre 0,65 y $1,87 \%$ anual respecto al valor medio de TMA del sitio (tabla 1).

Aun cuando no existan cambios en las precipitaciones totales, es posible que un incremento en la temperatura, como el observado en el $75 \%$ de las estaciones evaluadas, genere incrementos en la evapotranspiración y, como consecuencia, el balance hídrico sea menos

Tabla 1: Identificación (correspondencia con Figura 1), ubicación (departamento al que pertenecen y latitud/longitud), año de inicio de la serie, valores promedio de precipitaciones anuales (pp) y temperatura media anual (tma) con su coeficiente de variación indicado entre paréntesis y el porcentaje de cambio anual de ambas variables para cada estación meteorológica. Se indica con un * aquellas estaciones meteorológicas que muestran tendencias significativas en el período considerado. El porcentaje de cambio anual se obtuvo expresando el valor de la pendiente del análisis de tendencia como porcentaje del valor promedio de la variable (pp o tma). Se muestra el período histórico y el período 2000-2014. Datos elaborados para la presente edición.

\begin{tabular}{|c|c|c|c|c|c|c|c|c|c|c|}
\hline \multicolumn{3}{|c|}{ Estación meteorológica } & \multicolumn{4}{|c|}{ Histórico } & \multicolumn{4}{|c|}{$2000-2014$} \\
\hline$N^{\circ}$ & $\begin{array}{l}\text { Depto./año } \\
\text { de inicio de } \\
\text { la serie }\end{array}$ & $\begin{array}{l}\text { Lat/ } \\
\text { long }\end{array}$ & $\begin{array}{l}\text { pp media } \\
\text { anual } \\
(\mathrm{mm})\end{array}$ & $\begin{array}{c}\% \\
\text { cambio } \\
\text { anual } \\
\text { pp }\end{array}$ & $\begin{array}{l}\text { tma } \\
\left({ }^{\circ} \mathrm{C}\right)\end{array}$ & $\begin{array}{c}\% \\
\text { cambio } \\
\text { anual } \\
\text { tma }\end{array}$ & $\begin{array}{c}\text { pp media } \\
\text { anual (mm) }\end{array}$ & $\begin{array}{c}\% \\
\text { cambio } \\
\text { anual } \\
\text { pp }\end{array}$ & $\begin{array}{l}\text { tma } \\
\left({ }^{\circ} \mathrm{C}\right)\end{array}$ & $\begin{array}{c}\% \\
\text { cambio } \\
\text { anual } \\
\text { tma }\end{array}$ \\
\hline 1 & $\begin{array}{c}\text { Cushamen / } \\
1960\end{array}$ & $\begin{array}{c}-42,02 /- \\
71,14\end{array}$ & $\begin{array}{l}454,38 \\
(31,16)\end{array}$ & $-0,15$ & $\begin{array}{c}9,10 \\
(9,87)\end{array}$ & 0,11 * & $\begin{array}{l}470,78 \\
(21,44)\end{array}$ & 0,02 & $\begin{array}{c}9,18 \\
(5,93)\end{array}$ & 0,65 * \\
\hline 2 & $\begin{array}{c}\text { Cushamen / } \\
1960\end{array}$ & $\begin{array}{c}-42,1 /- \\
71,17\end{array}$ & & & $\begin{array}{c}9,67 \\
(6,04)\end{array}$ & 0,00 & & & $\begin{array}{c}9,61 \\
(15,32)\end{array}$ & $1,87^{*}$ \\
\hline 3 & $\begin{array}{c}\text { Cushamen / } \\
1960\end{array}$ & $\begin{array}{c}-42,5 /- \\
71,54\end{array}$ & $\begin{array}{l}899,58 \\
(34,15)\end{array}$ & $-1,17^{*}$ & $\begin{array}{c}9,71 \\
(11,64)\end{array}$ & 0,10 & $\begin{array}{c}552,87 \\
(38,7)\end{array}$ & $-4,11$ & $\begin{array}{c}9,29 \\
(11,72)\end{array}$ & 1,83 * \\
\hline 4 & $\begin{array}{c}\text { Cushamen / } \\
1960\end{array}$ & $\begin{array}{c}-42,61 /- \\
70,38\end{array}$ & $\begin{array}{l}120,91 \\
(51,9)\end{array}$ & $-0,05$ & & & $\begin{array}{l}102,51 \\
(66,7)\end{array}$ & $-1,92$ & & \\
\hline 5 & $\begin{array}{c}\text { Cushamen / } \\
1960\end{array}$ & $\begin{array}{c}-42,75 /- \\
71,01\end{array}$ & $\begin{array}{l}301,32 \\
(35,75)\end{array}$ & 0,06 & & & $\begin{array}{l}320,03 \\
(49,90)\end{array}$ & 2,49 & & \\
\hline 6 & $\begin{array}{c}\text { Futaleufú / } \\
1973\end{array}$ & $\begin{array}{c}-42,93 /- \\
71,15\end{array}$ & $\begin{array}{l}466,85 \\
(43,51)\end{array}$ & 2,09 * & $\begin{array}{c}8,85 \\
(6,73)\end{array}$ & $-0,11$ & $\begin{array}{l}619,63 \\
(31,45)\end{array}$ & $-0,80$ & $\begin{array}{c}8,74 \\
(6,08)\end{array}$ & 0,80 * \\
\hline 7 & $\begin{array}{c}\text { Futaleufú / } \\
1960\end{array}$ & $\begin{array}{c}-42,93 /- \\
71,27\end{array}$ & $\begin{array}{l}442,09 \\
(24,69)\end{array}$ & 0,21 & & & $\begin{array}{l}492,95 \\
(27,62)\end{array}$ & 4,09 * & & \\
\hline 8 & $\begin{array}{c}\text { Futaleufú / } \\
1964\end{array}$ & $\begin{array}{c}-42,97 /- \\
71,58\end{array}$ & & & $\begin{array}{l}11,33 \\
(19,3)\end{array}$ & $-0,26$ & $\begin{array}{l}1173,43 \\
(24,01)\end{array}$ & $-2,83^{*}$ & $\begin{array}{c}10,53 \\
(10,93)\end{array}$ & 1,52 * \\
\hline 9 & $\begin{array}{c}\text { Futaleufú / } \\
2000\end{array}$ & $\begin{array}{c}-43,08 /- \\
71,52\end{array}$ & & & & & $\begin{array}{l}1085,27 \\
(26,22)\end{array}$ & $-3,44$ & $\begin{array}{c}9,71 \\
(6,22)\end{array}$ & 0,62 \\
\hline 10 & $\begin{array}{c}\text { Futaleufú / } \\
1960\end{array}$ & $\begin{array}{c}-43,24 /- \\
71,36\end{array}$ & $\begin{array}{c}647,32 \\
(25,2)\end{array}$ & 0,02 & & & $\begin{array}{l}705,15 \\
(23,81)\end{array}$ & $-1,82$ & & \\
\hline 11 & $\begin{array}{c}\text { Futaleufú / } \\
1960\end{array}$ & $\begin{array}{c}-43,08 /- \\
71,25\end{array}$ & $\begin{array}{l}499,63 \\
(31,01)\end{array}$ & 0,60 * & & & $\begin{array}{l}629,83 \\
(29,94)\end{array}$ & $-0,80$ & & \\
\hline 12 & $\begin{array}{c}\text { Languiñeo / } \\
1960\end{array}$ & $\begin{array}{c}-43,68 /- \\
71,3\end{array}$ & $\begin{array}{l}563,20 \\
(27,15)\end{array}$ & $-0,16$ & $\begin{array}{c}8,07 \\
(9,18)\end{array}$ & 0,12 & $\begin{array}{l}556,45 \\
(27,41)\end{array}$ & $-2,10$ & $\begin{array}{c}8,12 \\
(10,51)\end{array}$ & 1,72 * \\
\hline 13 & $\begin{array}{c}\text { Tehuelches / } \\
2000\end{array}$ & $\begin{array}{c}-44,18 /- \\
70,56\end{array}$ & & & & & $\begin{array}{l}249,82 \\
(19,09)\end{array}$ & $-1,07$ & $\begin{array}{c}7,19 \\
(7,93) \\
\end{array}$ & 0,97 \\
\hline & & & & a & . & ivas & ,05). & & & \\
\hline
\end{tabular}


favorable, incrementando el estrés hídrico en algunos momentos del año. En el contexto del cambio climático existen evidencias que indican que incrementos en las temperaturas pueden generar, en algunos sistemas, un efecto de sequía con consecuencias negativas sobre la productividad (Zhao \& Running, 2010).

\section{Tendencia del NDVI-I de la serie 2000-2014 a partir de datos de MODIS}

En los departamentos Cushamen, Languiñeo y Tehuelches la mayoría de los píxeles mostró tendencias negativas de la NDVI-I, mientras que en el departamento Futaleufú la mayor parte mostró tendencias neutras, aunque con un valor elevado de píxeles con tendencias negativas. Asimismo, la ocurrencia de píxeles con tendencias positivas en todos los departamentos, fue muy pequeña y no mayor al 5\% (Figura 2). Al promediar el valor de la pendiente de la regresión entre los años y NDVI-I, expresada como porcentaje del valor inicial de la serie, de todos los pixeles de cada departamento se observaron tendencias decrecientes de 0,37; 0,$65 ; 0,76$ y $0,85 \%$ anual para los departamentos Futaleufú, Tehuelches, Cushamen y Languiñeo, respectivamente. Si consideramos los cuatro departamentos juntos los píxeles con tendencias negativas de la NDVI-I resultaron el 53,3\%, aquellos con tendencias neutras el $44,2 \%$ y aquellos con tendencias positivas, el 2,5\%. Esto debe representar una clara señal de alarma para aquellas personas involucradas en la toma de decisiones. Si bien es común la asociación entre precipitación y productividad tanto en el tiempo como en el espacio (Sala et al., 1988; 2012), en este trabajo no se puede argumentar que los cambios observados en el NDVI-I se deban solo a cambios en las precipitaciones. Por el contrario, de las variables climáticas analizadas en las diferentes estaciones meteorológicas, fue la temperatura, y no la precipitación, la que presentó tendencias que podrían explicar las tendencias observadas en la NDVI-I.

La mayor parte de los píxeles con tendencias negativas se concentraron en el centro-este de la región de estudio, dominando las tendencias neutras hacia el oeste (Figura 2). La concentración de píxeles con tendencias negativas en el centroeste del área pareciera indicar un patrón oeste-este asociado al gradiente de precipitación existente en la región. A futuro sería importante indagar con mayor precisión este patrón y relacionar los cambios con otras variables ambientales.

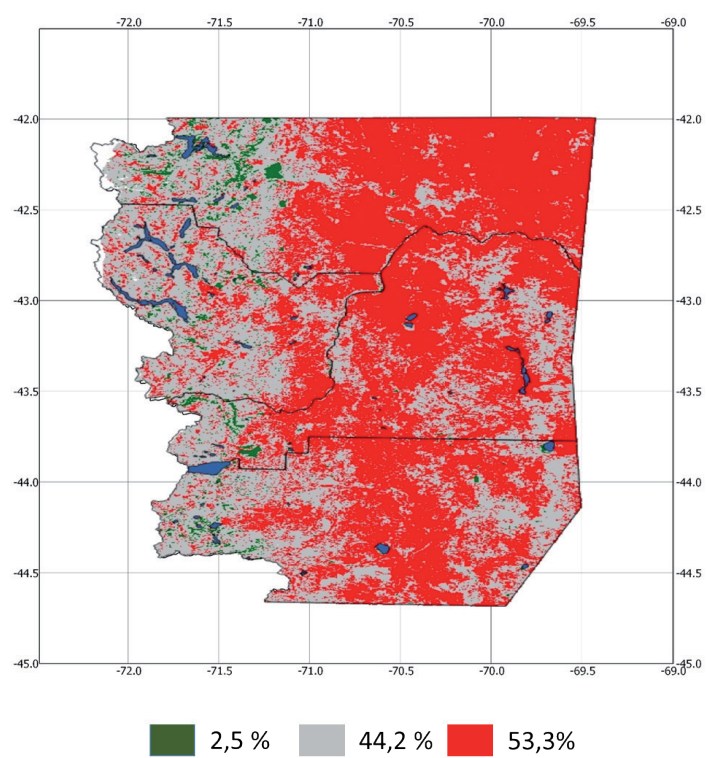

Figura 2: Tendencias de la NDVI-I para el área de estudio en el período 2000-2014. Se muestra además el porcentaje de píxeles con tendencias negativas (rojo), positivas (verde) o neutras (gris) de acuerdo al criterio mencionado en los materiales y métodos. En líneas negras se muestra el límite de los departamentos Cushamen, Futaleufú, Languiñeo y Tehuelches. En azul se indica la ubicación de espejos de agua. Datos elaborados para la presente edición.

\section{MARAS}

Para el conjunto de las 22 MARAS evaluadas, el tamaño de parches disminuyó en 15 de los casos evaluados (68\%); la cobertura vegetal disminuyó en $17(77 \%)$ y la cobertura forrajera disminuyó en 19 (86\%). A su vez, MP aumentó en 15 de los casos (68\%) y $\mathrm{M}$, en 11 (50\%) (Tabla 2$)$. Si analizamos los valores promedios de porcentaje de cambio anual de todas las MARAS para cada variable, en cobertura vegetal, cobertura forrajera y muerto en pie se observaron los mayores niveles de cambio anual, con valores de $-4,7,-5,6$ y $10,8 \%$ respectivamente.

Las plantas de estepa están adaptadas a estos ambientes secos con grandes variaciones climáticas interanuales y son capaces de permanecer latentes y rebrotar en años con condiciones favorables. Sin embargo, la sequía altera la disponibilidad de forraje habitual del campo y puede agravar las consecuencias de un pastoreo inadecuado en cuanto a momento, frecuencia e intensidad, pudiendo llegar a agotar las reservas de algunas plantas, provocando su muerte (Teague et al., 2013). El incremento mencionado en la cobertura de muerto en pie podría estar indicando una mortandad muy alta 
Tabla 2: Identificación (correspondencia con Figura 1), ubicación (departamento al que pertenecen y latitud/longitud) y porcentaje de cambio anual (\%/año) de indicadores de estado de la vegetación, tamaño de parche (TP), cobertura vegetal (CV), cobertura forrajera (CF), muerto en pie (MP) y mantillo (M). Los valores en la tabla indican la diferencia porcentual entre la primera y la segunda medición dividido por el número de años transcurridos entre ambas mediciones (fórmula 1).

\begin{tabular}{cccccccc}
\hline$N^{\circ}$ & Departamento & Lat/long & TP & CV & CF & MP & M \\
\hline A & Cushamen & $-42,54 /-69,73$ & $-0,7$ & $-9,0$ & $-11,4$ & 45,7 & $-10,6$ \\
B & Cushamen & $-42,20 /-70,02$ & $-8,8$ & $-3,3$ & $-3,5$ & $-9,1$ & 5,6 \\
C & Cushamen & $-42,21 /-70,02$ & $-3,2$ & $-8,1$ & $-10,8$ & $-5,8$ & 0,5 \\
D & Cushamen & $-42,01 /-70,45$ & $-5,6$ & $-7,7$ & $-12,4$ & 3,1 & $-0,6$ \\
E & Cushamen & $-42,34 /-70,50$ & $-1,8$ & 0,0 & $-1,6$ & 25,0 & 9,7 \\
F & Cushamen & $-42,36 /-70,52$ & $-2,8$ & $-13,4$ & $-5,8$ & 13,1 & $-6,8$ \\
G & Cushamen & $-42,35 /-70,53$ & $-2,6$ & $-5,9$ & $-4,3$ & 8,4 & 4,0 \\
H & Cushamen & $-42,82 /-70,59$ & $-3,8$ & $-8,8$ & $-8,6$ & 0,8 & $-6,9$ \\
I & Cushamen & $-42,54 /-70,75$ & 1,1 & $-8,8$ & $-8,9$ & 5,3 & 14,1 \\
J & Cushamen & $-42,08 /-70,85$ & $-3,2$ & $-3,3$ & $-4,0$ & 0,0 & $-2,9$ \\
K & Cushamen & $-42,07 /-70,88$ & $-6,1$ & $-5,4$ & $-6,4$ & 12,6 & 5,0 \\
L & Cushamen & $-42,07 /-70,88$ & 4,6 & 1,8 & 4,2 & $-3,1$ & $-0,8$ \\
M & Cushamen & $-42,80 /-70,96$ & $-2,2$ & $-8,0$ & $-9,8$ & 14,9 & $-10,1$ \\
N & Cushamen & $-42,03 /-71,01$ & 5,1 & $-1,4$ & $-1,0$ & 9,4 & 10,9 \\
O & Cushamen & $-42,03 /-71,01$ & 5,0 & $-3,4$ & $-3,1$ & 18,1 & 10,4 \\
P & Cushamen & $-42,41 /-71,10$ & $-3,8$ & 0,2 & $-12,3$ & 36,9 & $-6,7$ \\
Q & Futaleufu & $-42,94 /-71,17$ & 7,3 & 2,1 & 1,1 & 0,0 & $-2,2$ \\
R & Futaleufu & $-42,94 /-71,17$ & 16,3 & $-2,5$ & $-6,6$ & $-4,4$ & 10,4 \\
S & Languiñeo & $-42,80 /-69,58$ & $-7,5$ & $-11,9$ & $-11,9$ & $-16,0$ & 13,1 \\
T & Languiñeo & $-43,05 /-70,54$ & 1,9 & $-2,3$ & $-3,8$ & 15,2 & 10,8 \\
U & Tehuelches & $-44,38 /-70,50$ & $-8,4$ & $-10,6$ & $-14,9$ & 65,7 & 0,0 \\
V & Tehuelches & $-43,83 /-70,94$ & $-13,3$ & 6,3 & 12,3 & 1,5 & $-4,8$ \\
& PROMEDIO & & $-1,5$ & $-4,7$ & $-5,6$ & 10,8 & 1,9 \\
\hline
\end{tabular}

que puede llevar a los pastizales a una condición umbral de muy difícil retorno.

\section{Tendencias de las existencias ganaderas}

En el período 2005-2013 la tendencia de las existencias ganaderas en el total de los cuatro departamentos analizados fue significativamente $(p<0,05)$ negativa. Asimismo la tasa de disminución de animales fue de 66.024 UGOs al año, lo cual representa una disminución de 3\% anual referido al valor inicial de la serie.

Si bien no es posible realizar inferencias respecto a la heterogeneidad espacial de la tendencia observada en las existencias ganaderas dentro del área de estudio, su magnitud debe representar una luz de alarma para la región. La disminución del stock ganadero coincide con la caída anual de la NDVI-I, que entre 2000 y 2014 mantuvo una tasa negativa de 0,66\% anual. Esta caída en el NDVI-I posiblemente esté vinculada a disminuciones en la cantidad y calidad forrajera disponible para los animales, lo cual está fuertemente asociado al desempeño reproductivo de los animales (Texeira et al., 2012). En este sentido la disminución de las existencias ganaderas puede ser explicada por un ajuste decidido por los productores o bien debido a que las tasas reproductivas de las majadas y rodeos no son suficientes para mantener el stock en la región, provocando su disminución progresiva (Golluscio et al., 1998). Es posible que la conjunción de ambas razones esté explicando lo observado, por lo que estos resultados deben ser tenidos en cuenta al momento de fijar objetivos ganaderos tanto a escala predial como regional.

\section{Período histórico. Clima y existencias ganade- ras}

Si se considera el análisis histórico (19602014), en tres de las estaciones meteorológicas analizadas se observaron tendencias significativas en las precipitaciones. En dos estaciones se observaron tendencias positivas y sólo en una estación se observó una tendencia negativa. A partir del análisis de datos históricos de temperatura (1960-2014), sólo un caso muestra tendencia significativa, la cual fue positiva (Tabla 


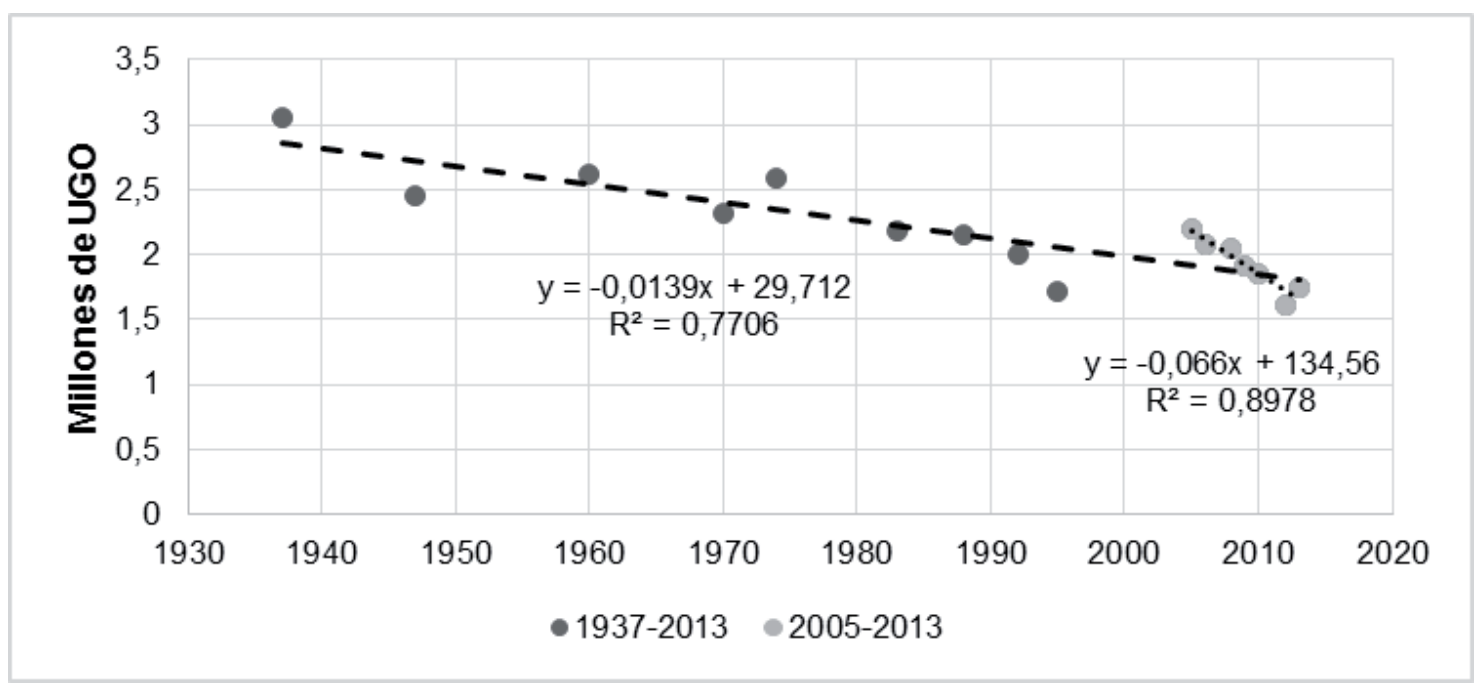

Figura 3: Existencias ganaderas por año para la suma de los cuatro departamentos del NO de Chubut considerados en este trabajo expresado en unidades ganaderas ovinas (UGO). Se muestran regresiones correspondientes a los períodos 1937-2013 y 2005-2013.

1). Estos resultados contrastan con el elevado porcentaje de estaciones meteorológicas (75\%) que mostraron incrementos de temperaturas en el período 2000-2014.

En lo que respecta a las existencias ganaderas, las tendencias fueron significativamente $(p<0,05)$ negativas para el período histórico (1937-2013). La tasa de disminución de animales fue de 13.860 UGOs al año para el período 1937-2013, lo cual representa una disminución de 0,45\% anual referido al valor inicial de la serie. Considerando los resultados mencionados en los párrafos anteriores es posible especular que el sistema ganadero sufre un proceso de deterioro histórico que en este trabajo queda registrado en la disminución de las existencias y de acuerdo a otros trabajos posee un correlato en el deterioro de los pastizales (Escobar, 1997; Perelman et al., 1997; Golluscio et al., 1998). Asimismo, se puede decir que el proceso se agravó en los últimos años con caídas más abruptas en el stock ganadero, lo cual coincide con incrementos en las temperaturas, deterioro de la estructura del pastizal y disminución de la productividad.

\section{CONCLUSIONES}

Los resultados de este trabajo permitieron realizar un diagnóstico de la evolución del clima, el pastizal y las existencias ganaderas en una amplia zona de Chubut para el período 20002014. En términos generales en el período mencionado se observan cambios en algunas variables climáticas, principalmente incrementos de temperatura, y tendencias negativas en lo que hace a la productividad y estructura del pastizal, lo cual podría ser responsable de la disminución del stock ganadero.

A partir de este trabajo surgen posibles líneas de investigación en lo referido al vínculo entre las variables analizadas y otras que se pudieran incorporar al análisis. Sería importante indagar en qué medida los cambios en temperatura pudieran tener un efecto en el balance hídrico, o si a pesar de no existir tendencias claras en las precipitaciones se observan o no cambios en su estacionalidad. Del mismo modo resultaría importante generar un sistema de monitoreo de la estructura y funcionamiento de la vegetación similar a los monitores MARAS para áreas de bosque y mallines.

Según informes de panoramas climáticos futuros como el desarrollado por Fundación Di Tella (2006), tendencias climáticas como las observadas en este trabajo se prolongarán en el tiempo, por lo que sería esperable que se profundicen los procesos de degradación. En los últimos años y con el fin de desacelerar las pérdidas de stock ganadero se difundió la suplementación estratégica, la implantación de pasturas, el mejoramiento de mallines, los ajustes de carga mediante evaluaciones forrajeras, la diversificación productiva y el monitoreo satelital de los recursos. Los resultados mostrados en este trabajo ponen en relieve la complejidad y fragilidad de los pastizales, y la gran importancia de realizar un uso sustentable basados en la planificación continua, información objetiva y considerando los diferentes elementos 
de su estructura y funcionamiento. Dichos criterios deben ser considerados no solo a escala predial sino también a escala regional.

\section{AGRADECIMIENTOS}

Los autores agradecen a la Subsecretaría de Recursos Hídricos de la Nación por la información climática disponible en su sitio web y al Ministerio de la Producción de la provincia del Chubut por la información de existencias ganaderas. A los productores ganaderos que permitieron instalar monitores MARAS en sus establecimientos y/o brindaron información meteorológica incorporada en este trabajo.

Anexo 1: Número de animales por año de cada especie para la suma de los cuatro departamentos del NO de Chubut considerados en este trabajo.

\begin{tabular}{ccccc}
\hline Año & Ovinos & Bovinos & Equinos & Caprinos \\
\hline 1937 & 1970349 & 84738 & 59389 & 109949 \\
1947 & 1673573 & 63201 & 41285 & 67748 \\
1960 & 1777506 & 60081 & 45119 & 123815 \\
1970 & 1750798 & 72537 & 7700 & 53939 \\
1974 & 1712785 & 90751 & 33151 & 57563 \\
1983 & 1612527 & 81974 & 7000 & 1600 \\
1988 & 1361652 & 83079 & 27204 & 61933 \\
1992 & 1247020 & 81610 & 24416 & 59670 \\
1995 & 954789 & 86944 & 21175 & 51212 \\
2005 & 1212104 & 118356 & 21734 & 78995 \\
2006 & 1105798 & 117796 & 20550 & 74485 \\
2008 & 1094603 & 115478 & 20510 & 70351 \\
2009 & 1020141 & 107409 & 18884 & 66870 \\
2010 & 954342 & 111357 & 19153 & 58383 \\
2012 & 782466 & 100220 & 18688 & 54177 \\
2013 & 977932 & 111722 & sd & 57993 \\
\hline
\end{tabular}

\section{BIBLIOGRAFÍA}

Ares, J.; A.M. Beeskow, M. Bertiller, M. Rostagno, M.P. Irisarri, J. Anchorena and G. Defossé, 1990. Structural and dynamic characteristics of overgrazed lands of northern Patagonia, Argentina. En: Breymeyer, A, editor. Managed grasslands. Elsevier science publishers, The Netherlands, pp. 149-175.

Barros, V.R.; B.V. Scian y H.F. Mattio, 1979. Campos de precipitación de la provincia de Chubut (período 1931-1960). Geoacta 10:175-192.

Bertiller, M.B.; J.O. Ares and A.J. Bisigato, 2002. Multiscale Indicators of Degradation in the Patagonian Monte, Argentina. Environmental Management. 30: 0704-0715.
Brown, J.H.; T.J. Valone and C.G. Curtin, 1997. Reorganization of an arid ecosystem in response to recent climate change. Proceedings of the National Academy of Sciences, 94, 18, 9729-9733.

Del Valle, H.F., 1998. Patagonian soils: a regional synthesis. Ecol. Aust. 8: 103-124.

Escobar, J., 1997. Desertización Chubut. Prodesar. Proyecto argentino alemán INTA/GTZ, demos un futuro a la Patagonia.

Fabricante, I.; M. Oesterheld and J.M. Paruelo, 2009. Annual and seasonal variation of NDVI explained by current and previous precipitation across Northern Patagonia. Journal of Arid Environments, 73(8): 745753.

Fundación Torcuato Di Tella e Instituto Torcuato Di Tella, 2006. Comunicación nacional de cambio climático: vulnerabilidad de la Patagonia y sur de las provincias de Buenos Aires y La Pampa. Informe final. 20 de abril de 2006.

Gaitán, J.J.; D. Bran, G. Oliva, G. Ciari, V. Nakamatsu, J. Salomone, D. Ferrante, G. Buono, V. Massara, G. Humano, D. Celdrán, W. Opazo and F.T. Maestre, 2013. Evaluating the performance of multiple remote sensing indices to predict the spatial variability of ecosystem structure and functioning in Patagonian steppes. Ecological Indicators 34: 181-191.

Gaitán, J.J.; D.D. Bran y C. Azcona, 2015. Tendencia del NDVI en el período 2000-2014 como indicador de la degradación de tierras en Argentina: ventajas y limitaciones. AGRISCIENTIA, 32(2): 83-93.

Golluscio, R.; A. Deregibus and J. Paruelo, 1998. Sustainability and management in the patagonian steppes. Ecología Austral 8: 265-284.

Golluscio, R. A.; A.T. Austin, G.C. García Martínez, M. Gonzalez-Polo, O. Sala, and R.B. Jackson, 2009. Sheep grazing decreases organic carbon and nitrogen pools in the Patagonian steppe: combination of direct and indirect effects. Ecosystems, 12(4): 686-697.

Iglesias, D., 2013. Análisis de la cadena de la carne ovina en Argentina. Estudios socioeconómicos de los sistemas agroalimentarios y agroindustriales $\mathrm{N}^{\circ} 11$. Ediciones INTA. 201 pp.

Jobbágy, E.G.; O.E. Sala and J.M. Paruelo, 2002. Patterns and controls of primary production in the Patagonian steppe: a remote sensing approach. Ecology, 83(2): 307-319.

Millennium Ecosystem Assessment, 2005. Ecosystems and Human Well-being: Synthesis. Island Press, Washington, DC. 137 pp.

Muller-Dombois, D.D.; H. Ellenberg, 1974. Aims and Methods of Vegetation Ecology. Wiley, New York, pp. 547.

Oliva, G.; J. Gaitán, D. Bran, V. Nakamatsu, J. Salomone, 
G. Buono and G. Ciari, 2009. MARAS: a monitoring system for Patagonian rangelands. Understanding Desertification and Land Degradation Trends, 22, 130.

Oliva, G; J.J. Gaitán, D. Bran, V. Nakamatsu, J. Salomone, G. Buono, J. Escobar, F. Frank, D. Ferrante, G. Humano, G. Ciari, D. Suarez y W. Opazo, 2011. Manual para la instalación y lectura de monitores MARAS (Monitoreo Ambiental para Regiones Áridas y Semiáridas). PNUD, Buenos Aires, Argentina. 74 pp.

Oñatibia, G.R. and M.R. Aguiar, 2016. Continuous moderate grazing management promotes biomass production in Patagonian arid rangelands. Journal of Arid Environments, 125, 73-79.

Paruelo, J.M.; H.P. Epstein, W.K. Lauenroth and I.C. Burke, 1997. ANPP estimates from NDVI for the Central Grassland Region of the US. Ecology 78: 953-958.

Paruelo, J. M.; E.G. Jobbágy, and O.E. Sala, 1998 a. Biozones of patagonia (Argentina). Ecología Austral, 8(2): 145-153.

Paruelo, J. M.; A. Beltran, E. Jobbágy, O.E. Sala and R.A. Golluscio, 1998 b. The climate of Patagonia: general patterns and controls on biotic. Ecol Austral, 8: 85-101.

Perelman, S.B.; R.J. Leon and J.P. Bussacca, 1997. Floristic changes related to grazing intensity in a Patagonian shrub steppe. Ecography 20: 400-406.

Sala, O.E.; W.J. Parton, L.A. Joyce and W.K. Lauenroth, 1988. Primary production of the central grassland region of the United States. Ecology 69: 40-45.

Sala, O.E.; L.A. Gherardi, L. Reichmann, E. Jobbágy, and D. Peters, 2012. Legacies of precipitation fluctuations on primary production: theory and data synthesis. Philosophical Transactions of the Royal Society B: Biological Sciences 367:3135-3144.
Soriano, A., 1956a. Aspectos ecológicos y pastoriles de la vegetación patagónica relacionados con su estado y capacidad de recuperación. Revista Investigaciones Agrícolas 10: 349-372.

Soriano, A., 1956b. Los distritos florísticos de la Provincia Patagónica. Revista de Investigaciones Agrícolas, 10(4): 323-347.

Teague, R.; F. Provenza, U. Kreuter, T. Steffens and M. Barnes, 2013. Multi-paddock grazing on rangelands: why the perceptual dichotomy between research results and rancher experience?. Journal of Environmental Management, 128: 699-717.

Texeira, M.; G. Baldi, and J. Paruelo, 2012. An exploration of direct and indirect drivers of herbivore reproductive performance in arid and semi arid rangelands by means of structural equation models. Journal of Arid Environments, 81: 26-34.

Tucker, C.J., 1979. Red and photographic infrared linear combinations for monitoring vegetation. Remote Sens. Environ 8: 127-150.

Tucker, C.J.; C.L. Vanpreat, M.J. Sharman, and G. Van Ittersum, 1985. Satellite remote sensing of total herbaceous biomass production in the Senegalese Sahel: 1980-1984. International Journal of Remote Sensing 7: 233-249.

Turner, D.P.; W.B. Cohen, K.S. Fassnacht and J.M. Briggs, 1999. Relationships between Leaf Area Index and Landsat TM Spectral Vegetation Indices across Three Temperate Zone Sites. Remote Sens. Environ 70: 5268.

Zhao, M. and S.W. Running, 2010. Drought-Induced Reduction in Global Terrestrial Net Primary Production from 2000 through 2009. Science. 329: 940-943. 